\title{
Validación del SCL-27 en población general y en población clínica argentina
}

\author{
Vanesa Góngora ${ }^{1}$ y Alejandro Castro Solano ${ }^{2}$
}

\begin{abstract}
RESUMEN
Este estudio tuvo como objetivo evaluar la adecuación del uso de la escala SCL-27 (Sympton Checklist-27) en población general y población clínica argentina y su comparación con el SCL-90R. Se utilizaron una muestra intencional de 400 personas de población general y una muestra de 85 pacientes en tratamiento psicológico ambulatorio. Se administraron el SCL-27, la escala Mental Health Continuum- Short Form, la escala de depresión CES-D, la escala de síntomas somáticos de Schaffer y la escala de Satisfacción con la vida. Los resultados indicaron que el SCL-27 muestra una altísima correlación en todas sus dimensiones con la escala original SCL-90-R. Presentó una alta validez convergente con medidas de depresión y de síntomas somáticos y moderada e inversa con medidas de bienestar. La fiabilidad fue adecuada en sus dimensiones y excelente en el Índice de Severidad Global. Esta escala mostró puntuaciones claramente diferenciadas entre población general y población clínica. Los resultados de este estudio permiten recomendar particularmente la utilización del Índice de Severidad Global del SCL-27 como medida general de trastornos psicológicos.
\end{abstract}

Palabras Clave: SCL-27, evaluación, psicopatología, validación.

\section{Validation of the SCL-27 in Argentinean general and clinical population}

\begin{abstract}
This study aimed to evaluate the adequacy of the SCL-27 scale (Sympton Checklist-27) to be used in Argentinean general and clinical populations and its comparison with the SCL90R. An intentional sample of 400 participants from general population and a sample of 85 patients attending outpatient psychological treatment were used. The SCL-27, the Mental Health Continuum-Short Form scale, the CES-D depression scale, the Schaffer somatic symptoms scale and the Satisfaction with life scale were administered. The results indicated that the SCL-27 shows a very high correlation in all its dimensions with the original SCL-90-R scale. It had a high convergent validity with measures of depression and somatic symptoms and moderate and inverse correlation with measures of well-being. The reliability was adequate in its dimensions and excellent in the Global Severity Index. The scale scores clearly differentiated between the general population and
\end{abstract}

${ }_{1}$ Consejo Nacional de Investigaciones Científicas y Técnicas (CONICET), Universidad de Palermo, Argentina; gongoravanesa@gmail.com; https://orcid.org/0000-0001-9048-5643

2 Consejo Nacional de Investigaciones Científicas y Técnicas (CONICET), Universidad de Palermo, Universidad de Buenos Aires, Argentina; alejandro.castrosolano@gmail.com; https://orcid.org/00000002-4639-3706

FINANCIAMIENTO | El presente proyecto fue realizado mediante los subsidios PIP CONICET $11220150100381 \mathrm{CO}$ "Hacia una aproximación émica de la psicología positiva. Los rasgos positivos como predictores del funcionamiento óptimo" y con subsidio UBACyT, 20020150100037BA "La evaluación de los rasgos de personalidad positivos. Su relación con los rasgos de personalidad patológicos (DSM5) y el bienestar psicológico". 
the clinical population. The results of this study let us recommend the use of the Global Severity Index of the SCL-27 as a general measure of psychological disorders.

Keywords: SCL-27, assessment, psychopathology, validation.

La evaluación de síntomas psicopatológicos es un área relevante dentro de la evaluación psicológica. Entre los instrumentos destinados a la detección de sintomatología clínica psicopatológica, el SCL-90-R (Symptoms Checklist 90-Revised) es uno de los más utilizados a nivel internacional (Derogatis, 1977). Este inventario permite detectar 9 categorias de sintomas: Somatizaciones, Obsesiones y compulsiones, Sensibilidad interpersonal, Depresión, Ansiedad, Hostilidad, Ansiedad fóbica, Ideación paranoide y Psicoticismo, y contar con un Índice de Severidad Global como medida general del malestar psicológico en el individuo. Puede ser utilizado en pacientes en tratamiento psicológico o psiquiátrico y en población general. Las propiedades psicométricas de este instrumento fueron estudiadas en distintos países, entre ellos Estados Unidos (Derogatis, 1977; Kevin Chapman, Petrie, \& Vines, 2012), Italia (Preti, Carta, \& Petretto, 2019; Prunas, Sarno, Preti, Madeddu, \& Perugini, 2012), Francia (Fortin, Coutu-Wakulczyk, \& Engelsmann, 1989), Alemania (Schmitz et al., 2000), Hungria (Urbán et al., 2014), China (Yu et al., 2019) y Ucrania (Sereda \& Dembitskyi, 2016) indicando adecuados niveles de fiabilidad y validez en muestras clínicas, de población general, rurales y de adolescentes (Casullo \& Castro Solano, 1999; Derogatis \& Unger, 2010; Fortin, et al., 1989; Kevin Chapman et al., 2012; Preti et al., 2019; Schmitz et al., 2000; Urbán et al., 2014; Yu et al., 2019). En Argentina, el SCL-90R ha demostrado una buena fiabilidad para todas las escalas $(a>.75)$ y evidencias de validez en población general y en población clínica (Casullo \& Castro Solano, 1999; Sanchez \& Ledesma, 2009). El inventario permitió diferenciar claramente entre población general y población clínica. En población clínica se encontraron diferencias de género significativas en las escalas de somatizaciones, sensibilidad interpersonal, depresión, ansiedad y en los indices generales (Sanchez \& Ledesma, 2009).

Sin embargo, más allá de las virtudes del SCL-90-R, una de sus mayores limitaciones se encuentra en la extensión de 90 ítems, lo que hace que sea poco práctica a la hora de realizar una detección rápida de síntomas. Por tal motivo, han surgido varias formas más breves del SCL-90-R que van de los 5 (Strand, Dalgard, Tambs, \& Rognerud, 2003) a los 53 items originales (Derogatis, 1993). Una mejor descripción de las distintas versiones breves del SCL-90-R y su comparación psicométrica puede encontrarse en Müller, Postert, Beyer, Furniss, y Achtergarde (2010) y en Prinz et al. (2013). De todas estas versiones, el SCL-27 es de los que mejor conserva las buenas propiedades psicométricas de la versión original, con el beneficio de contar con solo 27 ítems de la versión extensa (Müller et al., 2010; Prinz et al., 2013). Los items fueron seleccionados sobre la base del análisis factorial exploratorio del SCL-90-R en una muestra de validación y en pacientes con dolor crónico y reteniendo aquellos que presentaban altas correlaciones convergentes y bajas correlaciones discriminantes (Hardt \& Gerbershagen, 2001). Los 27 items se organizan en seis escalas de sintomas presentes en la última semana: sintomas depresivos, síntomas distímicos, síntomas vegetativos, sintomas agorafóbicos, sintomas de fobia social y síntomas de desconfianza. Además, 
permite obtener un Índice de Severidad Global (ISG) del malestar actual de la persona (Hardt \& Gerbershagen, 2001).

Diversos estudios han encontrado que el SCL-27 (Hardt \& Gerbershagen, 2001 ) presenta buenos niveles de fiabilidad (alfa >.70) para todas las escalas y el indice general de severidad en pacientes con trastornos afectivos $(n=2727)$ (Prinz et al., 2013), en pacientes con dolor crónico $(n=571)$ (Hardt \& Gerbershagen, 2001), en pacientes psiquiátricos $(n=449)$ (Kuhl et al., 2010), en un grupo de 100 madres de enfermos internados (Müller et al., 2010), en estudiantes universitarios ( $n=320)$ (Hardt, Dragan, \& Kappis, 2011) y en participantes de población general $(n=2780)$ (Hardt \& Gerbershagen, 2001). Esta versión presentó una alta correlación con la forma original de 90 ítems con correlaciones de $r=.87$ a $r=.98$ para sus escalas y para el índice de severidad en pacientes psiquiátricos y madres de pacientes internados (Kuhl et al., 2010; Müller et al., 2010). La estructura factorial del instrumento fue confirmada en población general (Hardt \& Gerbershagen, 2001), en pacientes psiquiátricos (Kuhl et al., 2010) y pacientes con trastornos afectivos (Prinz et al., 2013).

Por otro lado, el SCL-27 demostró buenos niveles de sensibilidad (98.41) y especificidad (94.59) para la detección de psicopatología (Hardt \& Gerbershagen, 2001; Müller et al., 2010) en pacientes con trastornos afectivos (Prinz et al., 2013). El instrumento también indicó buena sensibilidad al cambio (ISG $\mathrm{d}=.59$ ) en pacientes con trastornos afectivos luego de un tratamiento psiquiátrico comparable al obtenido en la versión de 90 items (ISG $d=.62$ ). Resultados similares se hallaron en pacientes con trastornos psiquiátricos (Kuhl et al., 2010) en los que las distintas subescalas del SC-27 evidenciaron una sensibilidad al cambio similar al SCL-90-R (ISG SCL-27 $r=$ .67; SCL-90-R $r=.75$ ).

En lo que se refiere a las diferencias individuales en las puntuaciones de esta escala, los resultados no han sido consistentes en las distintas investigaciones. No se hallaron diferencias de género en las dimensiones del SCL-27 en población de estudiantes alemanes y polacos (Hardt et al., 2011), en pacientes con dolor crónico (Hardt $\&$ Gerbershagen, 2001) y en población general (Hardt, Egle, \& Brähler, 2006). Sin embargo, Hardt et al. (2006) también reportaron en una segunda muestra de población general $(n=2179)$ que las mujeres puntuaban más alto que los hombres en todas las subescalas del SCL-27, menos en Desconfianza, en la que no había diferencias significativas. En relación con la edad, no se encontró evidencia de una asociación significativa en pacientes con dolor crónico (Hardt \& Gerbershagen, 2001) ni en una muestra de población general (Hardt et al., 2006), aunque en otra muestra también de población general se halló una relación inversa significativa en las escalas de Fobia Social y de Desconfianza (Hardt et al., 2006).

El SCL-27 ha sido evaluado en poblaciones de Alemania (Hardt et al., 2011; Hardt et al., 2006), Polonia (Hardt et al., 2011) y Suiza (Kuhl et al., 2010). Sin embargo, no se ha probado su utilidad en poblaciones de habla hispana. Este estudio tiene como objetivo evaluar la adecuación del uso de la escala SCL-27 en población general y población clínica argentina y su comparación con la versión original de 90 ítems. Para ello se examinarán las propiedades psicométricas del instrumento, se comparará las puntuaciones entre población clínica y no clínica y se establecerán diferencias individuales según edad, sexo y lugar de residencia. 


\section{MÉTODO}

\section{Participantes}

\section{Muestra población general}

Se utilizó una muestra intencional de 400 personas, 200 hombres y 200 mujeres, que tenian en promedio 40.44 años $(D E=14.12$, rango $=18-80$ años). El 12.8\% $(n=60)$ de la muestra eran extranjeros residentes en Argentina. El 66.7\% ( $n=267)$ de los participantes vivian en la Ciudad Autónoma de Buenos Aires y el $17.7 \%(n=71)$ en el Conurbano Bonaerense. El resto $(n=62,15.6 \%)$ residia en el interior del país. Con relación al nivel máximo de educación alcanzado, el 8.8\% $(n=35)$ había alcanzado el nivel básico de educación, el 48.2\% $(n=193)$ el nivel medio completo, el $37.8 \%(n=$ 151) un nivel terciario o universitario completo y un $5.2 \%(n=21)$ un nivel de postgrado completo. La mayoría de los participantes trabajaban $(n=359$, $81.9 \%)$, ubicándose principalmente en las categorias de empleado $(n=225$, $56.2 \%)$. El $25.4 \%(n=102)$ eran patrones, empleadores o trabajaban por su cuenta, un participante era trabajador, pero sin salario $(0.3 \%)$, y el resto de los participantes $(n=72,18.1 \%)$ no eran trabajadores activos (categoría que incluía a jubilados, desempleados y amas de casa). La mayoría de los participantes manifestó pertenecer a un nivel socioeconómico medio $(n=295$; $73.8 \%)$ y medio-alto $(n=44 ; 11 \%)$.

\section{Muestra clínica}

Se incluyeron 85 pacientes $(25$ hombres y 60 mujeres $)$ que se encontraban realizando en tratamiento psicológico ambulatorio en centros de salud mental de la ciudad de Buenos Aires. La edad media era de 40.83 años $(D E=16.0$, rango 18-75 años). El $58.8 \%(n=50)$ había sido diagnosticado con un trastorno de tipo ansioso, el $21.2 \%(n=18)$ con un trastorno de tipo depresivo, el $17.6 \%(n=15)$ con trastornos comórbidos con síntomas ansiosos y depresivos y un $2.4 \%(n=2)$ con algún otro trastorno. Se excluyeron a pacientes con diagnóstico de esquizofrenia, demencias o trastornos bipolares, así como aquellos cuyo estado de crisis les impedía completar los cuestionarios. En cuanto a la etapa de tratamiento, el $25.9 \%(n=22)$ de los pacientes se encontraban en la fase inicial, el $54.1 \%(n=46)$ en una etapa intermedia y el $20 \%(n=17)$ en una etapa final. La duración media de tratamiento de los participantes era de 6.75 meses $(\mathrm{DE}=8.59)$. Una minoría $(23.5 \%)$ había alcanzado la educación primaria $(n=20)$ como nivel máximo de estudios, $51.8 \%(n=44)$ la educación media y $24.7 \%(n=21)$ una educación terciaria o universitaria.

\section{Instrumentos}

SCL-27 (Symptom Checklist-27). Es un inventario de 27 items extraídos del SCL-90-R (Hardt \& Gerbershagen, 2001). Los items presentan diversos sintomas que una persona puede experimentar en la última semana. Tiene 5 opciones de respuesta de 0 (nada) a 4 (mucho). Los items se agrupan en 6 dimensiones: Sintomas Depresivos, Distimia, Sintomas Vegetativos, Agorafóbia, Fobia Social, y Desconfianza. El instrumento provee también un Índice de Severidad Global. Estudios han demostrado buenas evidencias de fiabilidad y validez del instrumento (Derogatis, 1977; Hardt et al., 2011; Kuhl et al., 2010; Müller et al., 2010; Prinz et al., 2013) así como su equivalencia 
con el SCL-90-R (Derogatis, 1977). Los ítems de esta versión se tomaron de la versión adaptada del SCL-90-R adaptada en Argentina (Casullo, 1998).

Mental Health Continuum- Short Form (MHC-SF). Esta escala evalúa el nivel de bienestar emocional, psicológico y social conforme a la teoría del bienestar de Keyes (Keyes, 2005). El bienestar emocional comprende el afecto positivo y satisfacción con la vida (bienestar hedónico); el bienestar psicológico comprende la autonomía, el control, el crecimiento personal, las relaciones personales, la autoaceptación y el propósito en la vida; en tanto que el bienestar social abarca la aceptación, la actualización, la contribución social, la coherencia y la integración social. Está conformada por 14 ítems con seis opciones de respuesta de 0 (nunca) a 5 (todos los dias). Estudios internacionales han confirmado su estructura factorial, asi como han demostrado buenas evidencias de validez convergente, discriminante $\mathrm{y}$ fiabilidad (e.g., Gallagher, Lopez, \& Preacher, 2009; Joshanloo, Wissing, Khumalo, \& Lamers, 2013; Karaś, Cieciuch, \& Keyes, 2014). Este instrumento se encuentra validado en Argentina (Lupano Perugini, de la Iglesia, Castro Solano, \& Keyes, 2017). La fiabilidad por escala para esta muestra fue bienestar emocional: $\mathrm{a}=.86$; bienestar social: $\mathrm{a}=.77$; bienestar psicológico: $\mathrm{a}$ $=.79$; bienestar total: $\mathrm{a}=.87$.

Escala de depresión CES-D. Esta escala fue diseñada para la detección de sintomatología depresiva en estudios epidemiológicos con población general adulta (Radloff, 1977). Consta de 20 ítems con 4 opciones de respuesta de 1 (menos de 1 día) a 4 (5 a 7 dias). La escala ha sido adaptada en Argentina mostrando alta consistencia interna, estabilidad temporal y validez convergente con diversas pruebas que evalúan sintomatología depresiva (Casullo, 2004). En esta muestra la consistencia interna para la escala fue $\alpha=$ .85 .

Escala de sintomas somáticos de Schaffer (Somatic Symptoms Index). Es una escala que evalúa la severidad de sintomas somáticos (Schafer \& Ferraro, 2013). Consta de 10 ítems referidos a la experimentación de distintos sintomas somáticos durante los últimos 30 días. Posee seis opciones de respuesta que van desde 1 (nada) a 6 (casi todos los dias). La consistencia interna de la escala en esta muestra es $\alpha=.82$.

Escala de Satisfacción con la vida (SWLS). Es una escala que evalúa el grado de satisfacción global con la vida (Diener, Emmons, Larsen, \& Griffin, 1985). Está conformada por cinco ítems que se responden a través de 7 opciones de respuesta tipo Likert desde 1 (muy en desacuerdo) a 7 (muy de acuerdo). Las buenas propiedades psicométricas de esta escala han sido avaladas con numerosos estudios internacionales (Diener et al., 1985; Pavot, Diener, Colvin, \& Sandvik, 1991), siendo una de las más utilizadas en las investigaciones como medida del bienestar en tanto componente cognitivo de la satisfacción (Diener et al., 1985; Pavot \& Diener, 2009). Esta escala se encuentra validada en población argentina (Castro Solano \& Diaz Morales, 2000). En esta muestra el nivel de consistencia interna es $\alpha=.88$.

\section{Procedimiento}

Esta investigación cumple los estándares éticos de la American Psychological Association (2010). Los participantes de las dos muestras fueron informados de los propósitos de la investigación, la confidencialidad de los datos y de su derecho a rehusarse a participar y retirarse de la investigación 
cuando lo deseasen. Los participantes de las dos muestras firmaron el consentimiento informado.

En la muestra de población general, las administraciones fueron realizadas por estudiantes de la carrera de Psicología de la Universidad de Palermo bajo la supervisión de los autores de este trabajo. Para la muestra clínica, se solicitó a terapeutas que seleccionen a pacientes en distintas etapas de tratamiento que pudieran participar en el estudio conforme a los criterios de inclusión y exclusión mencionados en Participantes. La administración de las pruebas se llevó a cabo en presencia de un miembro del equipo de investigación. En las dos muestras, la recolección de datos se efectuó en forma individual en una sesión de aproximadamente 45 minutos. El análisis de los datos se realizó con el programa estadístico SPSS 18.

\section{RESULTADOS}

\section{Correlaciones SCL-27 / SCL-90-R}

En primer lugar, se calcularon las puntuaciones para cada una de las escalas de las dos pruebas, siguiendo los criterios establecidos por los autores. Posteriormente, se comparó la asociación entre las dimensiones equivalentes en cada instrumento, por ejemplo, Sintomas Depresivos del SCL-27 y Depresión del SCL-90-R, utilizando el coeficiente de correlación de Pearson. Este procedimiento se realizó con la muestra de población general y con la muestra clínica. Los resultados se presentan en la tabla 1. Las correlaciones, tanto de las dimensiones de los dos instrumentos como del índice de severidad global, son positivas, altas y significativas, siendo muy similares en las dos muestras de estudio.

Tabla 1.

Correlaciones del SCL-27 con el SCL-90R en una población general y clínica.

\begin{tabular}{lcc}
\hline Dimensiones SCL-27 / Dimensiones SCL-90-R & $\begin{array}{c}\text { General } \\
(n=400) \\
\boldsymbol{r}\end{array}$ & $\begin{array}{c}\text { Clínica } \\
(n=85) \\
\boldsymbol{r}\end{array}$ \\
\hline Síntomas Depresivos / Depresión & .83 & .85 \\
Agorafobia / Ansiedad Fóbica & .91 & .88 \\
Distimia / Obsesiones y Compulsiones & .85 & .87 \\
Síntomas Vegetativos / Somatizaciones & .89 & .89 \\
Fobia Social / Sensitividad Interpersonal & .90 & .92 \\
Desconfianza / Ideación Paranoide & .96 & .96 \\
Índice de Severidad Global & .97 & .97 \\
\hline
\end{tabular}

En segundo lugar, se calcularon los valores de fiabilidad utilizando el coeficiente alfa de consistencia interna para las dimensiones del SCL-27. En la tabla 2, se presenta su comparación con los valores obtenidos en las dimensiones equivalentes en el SCL-90-R para cada muestra. Los valores del SCL-27 son algo más bajos que los obtenidos para el SCL-90-R, a excepción del Índice de Severidad Global. Debe tenerse en cuenta que la cantidad de ítems en cada dimensión del SCL-27 es, en general, menos de la mitad que en la versión original. Los valores para la escala Distimia se encuentra algo bajos en relación con lo considerado adecuado para la consistencia interna de las escalas (Nunnally \& Bernstein, 1994). Por otro lado, en las dimensiones Agorafobia, Distimia, Sintomas Vegetativos y Fobia Social se observa una mayor consistencia interna en la muestra clínica que en la de población 
general. La muestra clínica, en términos generales, presenta mayor fiabilidad en sus dimensiones.

Tabla 2.

Consistencia interna de las escalas del SCL-27 y del SCL-90-R.

\begin{tabular}{lcccc}
\hline & \multicolumn{2}{c}{$\begin{array}{c}\text { General } \\
(n=400)\end{array}$} & \multicolumn{2}{c}{$\begin{array}{c}\text { Clínica } \\
(n=85)\end{array}$} \\
\cline { 2 - 5 } Dimensiones SCL-27 / Dimensiones SCL-90-R & $\begin{array}{c}\text { SCL-27 } \\
\text { SCL-90-R }\end{array}$ & $\begin{array}{c}\text { SCL-27 } \\
\mathbf{\alpha}\end{array}$ & $\begin{array}{c}\text { SCL-90-R } \\
\mathbf{\alpha}\end{array}$ \\
\hline Síntomas Depresivos / Depresión & .72 & .88 & .70 & .88 \\
Agorafobia / Ansiedad Fóbica & .76 & .80 & .84 & .84 \\
Distimia / Obsesiones y Compulsiones & .68 & .84 & .74 & .84 \\
Síntomas Vegetativos / Somatizaciones & .76 & .85 & 82 & .88 \\
Fobia Social / Sensibilidad Interpersonal & .75 & .82 & .84 & .86 \\
Desconfianza / Ideación Paranoide & .73 & .78 & .75 & .79 \\
Índice de Severidad Global & .92 & .97 & .93 & .97 \\
\hline
\end{tabular}

\section{Validez de Criterio}

Para evaluar la validez de criterio, se calcularon con la muestra de población general las correlaciones de las distintas dimensiones del SCL-27 y el bienestar emocional, psicológico y social (MHC-SF), el nivel de depresión (CES-D), los sintomas somáticos (SCHAFFER) y la satisfacción con la vida (SWLS). En la tabla 3 se presentan los resultados. Se encontraron correlaciones inversas, significativas y moderadas entre las dimensiones del SCL-27 y todos los tipos de bienestar, registrándose las correlaciones más altas con el bienestar emocional, psicológico y total. De igual forma, se hallaron correlaciones inversas de bajas a moderadas con la satisfacción con la vida. Con respecto a otras escalas de sintomas, los resultados mostraron correlaciones directas de moderadas a fuertes con los síntomas somáticos, y fuertes con los sintomas depresivos.

Tabla 3.

Correlaciones del SCL-27 y el MH-SF, CES-D, Schaffer y SWLS $(n=400)$.

\begin{tabular}{|c|c|c|c|c|c|c|c|}
\hline & B.Emoc. & B.Psico & B.Social & B.Total & CESD & Schaffer & SWLS \\
\hline & $r$ & $r$ & $r$ & $r$ & $r$ & $r$ & $r$ \\
\hline Síntomas Depresivos & $-.40^{\star * \star}$ & $-.35^{* \star *}$ & $-.15^{\star *}$ & $-.37^{\star \star \star}$ & $.61^{* * *}$ & $.34^{* * *}$ & $-.30^{\star * \star}$ \\
\hline Agorafobia & $-.24^{\star \star \star}$ & $-.24^{* \star \star}$ & -.02 & $-.20^{\star \star \star}$ & $.43^{* \star *}$ & $.32^{* \star *}$ & $-.14^{\star \star}$ \\
\hline Distimia & -.05 & $-.30^{* * *}$ & $-.28^{\star \star \star}$ & $-.42^{\star \star \star}$ & $.50^{* * *}$ & $.36^{\star \star \star}$ & $-.17^{\star \star \star}$ \\
\hline Síntomas Vegetativos & $-.23^{\star \star \star}$ & $-.24^{\star \star \star}$ & $-.10^{*}$ & $-.23^{\star \star \star}$ & $.43^{* \star *}$ & $.50^{\star \star *}$ & $-.18^{\star \star \star}$ \\
\hline Fobia Social & $-.29^{* \star \star}$ & $-.34^{\star * *}$ & $-.13^{\star *}$ & $-.31^{* \star *}$ & $.45^{* \star *}$ & $.35^{\star \star *}$ & $-.21^{\star \star *}$ \\
\hline Desconfianza & $-.29^{* \star \star}$ & $-.31^{\star \star \star}$ & $-.20^{\star \star *}$ & $-.33^{* \star *}$ & $.52^{\star \star \star}$ & $.43^{\star \star *}$ & $-.20^{\star * *}$ \\
\hline Îndice de Severidad Global & $-.31^{* \star \star \star}$ & $-.38^{* \star \star x}$ & $-.19^{* \star \star}$ & $-.39^{* * \star}$ & $.62^{\star \star *}$ & $.50^{* * \star}$ & $-.25^{* \star \star}$ \\
\hline
\end{tabular}

Nota: B.Emoc. $=$ Bienestar Emocional; B.Psico = Bienestar Psicológico; B.Social = Bienestar Total; B. Total $=$ Bienestar Total. ${ }^{*} p$ $<.05 ;{ }^{* *} p<.01 ;{ }^{* *} p<.001$.

\section{Comparación de las puntuaciones del SCL-27 entre población general y población clínica}

Posteriormente, se compararon las puntuaciones en las dimensiones del SCL-27 y del Índice de Severidad Global entre la muestra de población general 
y la de población clínica. Se utilizó la prueba t para muestras independientes. Los resultados se presentan en la tabla 4. Todas las medias en la muestra clínica resultan más altas que en la muestra de población general. El grupo clínico presenta mayores Síntomas Depresivos, Agorafobia, Sintomas Vegetativos, Desconfianza y el Índice de Severidad Global que el grupo de población general, aunque con un tamaño de efecto pequeño.

Tabla 4.

Comparación del SCL-27 entre población general y población clínica.

\begin{tabular}{|c|c|c|c|c|}
\hline SCL-27 dimensiones & $\begin{array}{c}\text { General } \\
(n=400) \\
M(D E)\end{array}$ & $\begin{array}{c}\text { Clínica } \\
(n=85) \\
M(D E)\end{array}$ & $t$ & $d$ \\
\hline Síntomas Depresivos & $.49(.67)$ & $.76(.72)$ & $-3.05^{* *}$ & .40 \\
\hline Agorafobia & $.40(.56)$ & $.63(.79)$ & $-2.29^{*}$ & .30 \\
\hline Distimia & $.89(.75)$ & $.91(.79)$ & -0.25 & .03 \\
\hline Síntomas Vegetativos & $.47(.62)$ & $.69(.74)$ & $-2.64^{*}$ & .35 \\
\hline Fobia Social & $.53(.67)$ & $.75(.89)$ & -1.97 & .26 \\
\hline Desconfianza & $.63(.74)$ & $.88(.85)$ & $-2.31^{*}$ & .30 \\
\hline Índice de Severidad Global & $.55(.53)$ & $.76(.62)$ & $-2.90^{*}$ & .38 \\
\hline
\end{tabular}

\section{Diferencias Individuales en las dimensiones del SCL-27 por variables demográficas: sexo, edad, lugar de residencia, nivel educativo}

En primer lugar, se calculó la prueba t para muestras independientes para las dimensiones del SCL-27 utilizando la variable sexo como criterio. En la muestra de población general no se encontraron diferencias en ninguna dimensión, ni en el Índice de Severidad Global entre hombres y mujeres. En cambio, en la muestra de población clínica, las mujeres puntuaron significativamente más alto en Sintomas Vegetativos $(t=-2.81, p<.01, d=$ $.67)$, Distimia $(t=-2.77, p<.01, d=.66)$, Depresión $(t=-3.74, p<.001, d$ $=.89) \mathrm{y}$ en el Índice de Severidad Global $(t=-2.65, p<.01, d=.63)$.

Para analizar la asociación entre el SCL-27 y la edad de los participantes, se realizaron correlaciones de Pearson con cada dimensión del instrumento. No se encontraron asociaciones estadísticamente significativas entre ninguna dimensión de la escala y la edad en la muestra de población general. Sin embargo, se hallaron asociaciones inversas entre edad y las dimensiones Agorafobia $(r=-.40, p<.001)$, Fobia Social $(r=-.33, p<.01)$ y el Índice de Severidad Global $(r=-.27, p<.05)$ en la muestra clínica.

Por otro lado, se calcularon las diferencias en las puntuaciones del SCL27 por nivel educativo a través del coeficiente de correlación de Pearson. No se hallaron correlaciones estadisticamente significativas entre las dimensiones del SCL-27 y el nivel educativo en la muestra de población general. Sin embargo, se encontró una asociación inversa moderada entre Sintomas Vegetativos y nivel educativo $(r=-.32, p<.01)$ en la muestra clínica.

Finalmente, se computaron pruebas ANOVA de un factor para evaluar diferencias en las dimensiones del SCL-27 por lugar de residencia (CABA/Gran Buenos Aires/Interior). No se hallaron diferencias estadísticamente significativas en ninguna de las dimensiones del SCL-27 ni en el Índice se Severidad Global, tanto por lugar de residencia en la muestra de población general ni en la muestra clínica. 


\section{Puntajes de corte para considerar riesgo de sintomatología}

Se calcularon los puntajes $\mathrm{T}$ normalizados, tal como se muestra en la tabla 5, para las puntuaciones directas del Índice de Severidad Global (ISG) para la muestra de hombres y de mujeres de población general. El instrumento establece que un puntaje $\mathrm{T}$ superior a 63 indica riesgo de psicopatología.

Tabla 5 .

Puntajes T normalizados para el Índice de Severidad Global del SCL-27.

\begin{tabular}{ccc}
\hline Puntaje T & ISG Hombres & ISG Mujeres \\
\hline T30 & .01 & .01 \\
T40 & .07 & .07 \\
T50 & .35 & .46 \\
T60 & 1.06 & 1.18 \\
T63 & 1.29 & 1.40 \\
T65 & 1.51 & 1.66 \\
T70 & 1.73 & 1.99 \\
T75 & 2.47 & 2.18 \\
\hline
\end{tabular}

\section{DISCUSIÓN}

Este estudio ha examinado la adecuación del SCL-27 (Hardt \& Gerbershagen, 2001) para su utilización en el contexto argentino. Esta escala abreviada muestra una altísima correlación en todas sus dimensiones con la escala original SCL-90R (Derogatis, 1977), en consonancia con todos los estudios previos que compararon los dos instrumentos (Kuhl et al., 2010; Müller et al., 2010).

Con respecto a las evidencias de fiabilidad y validez, el SCL-27 (Hardt \& Gerbershagen, 2001) presenta una adecuada fiabilidad, teniendo en cuenta la reducida cantidad de items en sus dimensiones, similar a los estudios previos (Hardt et al., 2011; Hardt \& Gerbershagen, 2001; Müller et al., 2010; Prinz et al., 2013). En la muestra de población clínica, los niveles de consistencia interna han sido más elevados, posiblemente por una mayor homogenización de la muestra. Hay que tener en cuenta que es un instrumento de rastrillaje clínico, y en población no consultante puede haber gente con y sin sintomatologia, en tanto que en la muestra clínica ya tienen un diagnóstico clínico. Los estudios previos con población clínica también mostraron mejores niveles de consistencia interna que los que emplearon población no clínica (Kuhl et al., 2010; Prinz et al., 2013). No obstante, en el Índice de Severidad Global, la consistencia interna es excelente y similar en las dos muestras empleadas.

El SCL-27 (Hardt \& Gerbershagen, 2001) ha demostrado una alta validez convergente con medidas de depresión y de síntomas somáticos, y moderada e inversa con medidas de bienestar. Esto es coherente con los estudios previos con el instrumento y medidas de depresión (Prinz, et al., 2013). Debe tenerse en cuenta que es el primer estudio en incluir medidas de bienestar para evaluar la validez convergente.

Esta escala presenta puntuaciones claramente diferenciadas entre población general y población clínica, particularmente en síntomas depresivos y somáticos, así como en su índice global de severidad, lo que permite 
sostener su utilización para diferenciar personas con y sin psicopatología (Hardt \& Gerbershagen, 2001).

En cuanto a las diferencias individuales por variables sociodemográficas, no se encontraron diferencias significativas en población general. No obstante, las mujeres de la muestra clínica mostraron más sintomas ansiosos, depresivos, distímicos y de severidad global que sus pares masculinos. Resultados similares se hallaron en el estudio realizado con el SCL-90-R en Argentina (Sanchez \& Ledesma, 2009). Las mujeres han reportado sistemáticamente mayores niveles de sintomatologia en el SCL-90-R (Casullo \& Castro Solano, 1999; Derogatis, 1977; Sanchez \& Ledesma, 2009; Sereda \& Dembitskyi, 2016). Sin embargo, estos resultados no han sido consistentes en los estudios con el SCL-27 y solo uno encontró diferencias por género (Hardt et al., 2006).

Los pacientes con menor edad presentaban mayores síntomas de agorafobia, fobia social y severidad que los pacientes de mayor edad. Esta asociación inversa ha sido también reportada por Hardt et al., (2006), aunque no ha sido un hallazgo replicable en otras muestras (Hardt et al., 2006; Hardt \& Gerbershagen, 2001).

Por último, en población clínica se halló que un menor nivel educativo se asociaba con mayores sintomas vegetativos, si bien esta asociación ha sido leve. Aunque no hay hasta el momento investigaciones con el SCL-27 (Hardt \& Gerbershagen, 2001) y el nivel educativo, estudios realizados con el SCL-90-R también han reportado esta asociación (Sayar, Kirmayer, \& Taillefer, 2003; Sohn, Kim, Ryu, Oh, \& Ahn, 2010).

Los resultados de este estudio permiten recomendar particularmente la utilización del Indice de Severidad Global como medida general de trastornos psicológicos, teniendo en cuenta: la excelente correlación con la medida equivalente del SCL-90-R (Derogatis, 1977), la alta consistencia interna, la buena validez convergente y la capacidad de diferenciar población clínica de no clínica. Asimismo, este estudio provee puntuaciones T equivalentes para su empleo en la detección de población en riesgo en la evaluación psicológica.

Dentro de las limitaciones de esta investigación pueden mencionarse el tamaño relativamente pequeño de la muestra clínica, su conformación mayoritaria femenina y con sintomatología fundamentalmente depresivoansiosa. De todas formas, estas constituyen el tipo de patologías que se relacionan más con las dimensiones que evalúa el SCL-27 (Hardt \& Gerbershagen, 2001). Asimismo, tanto la muestra clínica como la de población general pertenecen a la región metropolitana de Buenos Aires. Futuros estudios deberán evaluar su aplicación en otras regiones de Argentina y de otros países de habla hispana y contrastar la evaluación de esta escala con criterios clinicos o entrevistas estructuradas.

En resumen, el SCL-27 (Hardt \& Gerbershagen, 2001) es una escala abreviada que resulta recomendable, válida y fiable para utilizarse en el rastrillaje de sintomatología clínica en población argentina a través de su indice de severidad global, siendo equivalente a las propiedades psicométricas obtenidas en el SCL-90-R (Derogatis, 1977).

\section{REFERENCIAS}

American Psychological Association (2010). Ethical Principles of Psychologists and Code of Conduct. Recuperado de https://www.apa.org/ethics/code/

Castro Solano, A. \& Diaz Morales, J. (2000). Estilos de personalidad, objetivos de vida y satisfacción vital. 
En M. P. Sanchez Lopez \& M. M. Casullo (Eds.), Los estilos de personalidad. Una perspectiva Iberoamericana (pp. 195-231). Madrid: Miño y Dávila.

Casullo, M. M. (1998). El inventario SCL-90-R. Buenos Aires: Departamento de Publicaciones. Facultad de Psicología. Universidad de Buenos Aires.

Casullo, M. M. (2004). Sintomas psicopatológicos en adultos urbanos. Psicología y Ciencia Social, 6(1), 4957.

Casullo, M. M. \& Castro Solano, A. (1999). Sintomas psicopatológicos en estudiantes adolescentes argentinos, aportaciones del SCL-90-R . Anuario de Publicaciones, 147-157.

Derogatis, L. (1977). SCL-90-R Administration, Scoring and Procedures Manual - II. Towson, MD: Clinical Psycometric Research.

Derogatis, L. (1993). Brief Symptom Inventory (BSI) administration, scoring, and procedures manual. Minneapolis, MN: NCS Pearson. Inc.

Derogatis, L. \& Unger, R. (2010). Symptom Checklist-90-Revised. En I. B. Weiner \& W. E. Craighead (Eds.), The Corsini Encyclopedia of Psychology (pp. 1-2). New Jersey: Wiley \& Sons. https://doi.org/10.1002/9780470479216.corpsy0970

Diener, E., Emmons, R. A., Larsen, R. J., \& Griffin, S. (1985). The Satisfaction With Life Scale. Journal of Personality Assessment, 49(1), 71-75. https://doi.org/10.1207/s15327752jpa4901_13

Fortin, M. F., Coutu-Wakulczyk, G., \& Engelsmann, F. (1989). Contribution to the validation of the SCL-90$\mathrm{R}$ in French-speaking women. Health Care for Women International, 10(1), $27-41$. https://doi.org/10.1080/07399338909515836

Gallagher, M. W., Lopez, S. J., \& Preacher, K. J. (2009). The Hierarchical Structure of Well-Being. Journal of personality, 77(4), 1025-1050. https://doi.org/10.1111/j.1467-6494.2009.00573.x

Hardt, J., Dragan, M., \& Kappis, B. (2011). A short screening instrument for mental health problems: The Symptom Checklist-27 (SCL-27) in Poland and Germany. International journal of psychiatry in clinical practice, 15(1), 42-49. https://doi.org/10.3109/13651501.2010.523791

Hardt, J., Egle, U. T., \& Brähler, E. (2006). Die Symptom-Checkliste-27 in Deutschland [The symptom checklist-27 in Germany]. Psychotherapie, Psychosomatik, medizinische Psychologie, 56(7), $276-284$. https://doi.org/10.1055/s-2006-932577

Hardt, J., \& Gerbershagen, H. U. (2001). Cross-validation of the SCL-27: a short psychometric screening instrument for chronic pain patients. European Journal of Pain, 5(2), 187-197. https://doi.org/10.1053/eujp.2001.0231

Joshanloo, M., Wissing, M. P., Khumalo, I. P., \& Lamers, S. M. A. (2013). Measurement invariance of the Mental Health Continuum-Short Form (MHC-SF) across three cultural groups. Personality and Individual Differences, 55(7), 755-759. https://doi.org/10.1016/j.paid.2013.06.002

Karaś, D., Cieciuch, J., \& Keyes, C. L. M. (2014). The Polish adaptation of the Mental Health ContinuumShort Form (MHC-SF). Personality and Individual Differences, 69(0), $104-109$. https://doi.org/10.1016/j.paid.2014.05.011

Kevin Chapman, L., Petrie, J., \& Vines, L. (2012). Factor structure analysis of the SCL-90-R in a community-based sample of African American women. Psychiatry Research, 199(2), $140-144$. https://doi.org/10.1016/j.psychres.2012.03.028

Keyes, C. L. M. (2005). Mental illness and/or mental health? Investigating axioms of the complete state model of health. Journal of consulting and clinical psychology, 73(3), 539-548. https://doi.org/10.1037/0022-006X.73.3.539

Kuhl, H. C., Hartwig, I., Petitjean, S., Müller-Spahn, F., Margraf, J., \& Bader, K. (2010). Validation of the Symptom Checklist SCL-27 in psychiatric patients: Psychometric testing of a multidimensional short form. International Journal of Psychiatry in Clinical Practice, 14(2), $145-149$. https://doi.org/10.3109/13651501003660484

Lupano Perugini, M. L., de la Iglesia, G., Castro Solano, A., \& Keyes, C. L. M. (2017). The Mental Health Continuum-Short Form (MHC-SF) in the Argentinean Context: Confirmatory Factor Analysis and Measurement Invariance. Europe's Journal of Psychology, 13(1), 93-108. https://doi.org/10.5964/ejop.v13i1.1163

Müller, J. M., Postert, C., Beyer, T., Furniss, T., \& Achtergarde, S. (2010). Comparison of Eleven Short Versions of the Symptom Checklist 90-Revised (SCL-90-R) for Use in the Assessment of General Psychopathology. Journal of Psychopathology and Behavioral Assessment, 32(2), 246-254. https://doi.org/10.1007/s10862-009-9141-5

Nunnally, J. C. \& Bernstein, I. H. (1994). Psychometric theory. New York: McGraw-Hill.

Pavot, W. \& Diener, E. (2009). Review of the Satisfaction With Life Scale. En E. Diener (Ed.), Assessing wellbeing: The collected works of Ed Diener (pp. 101-117). New York, NY: Springer Science + Business Media. https://doi.org/10.1007/978-90-481-2354-4 5

Pavot, W., Diener, E., Colvin, C. R., \& Sandvik, E. (1991). Further validation of the Satisfaction With Life Scale: Evidence for the cross-method convergence of well-being measures. Journal of Personality Assessment, 57(1), 149-161. https://doi.org/10.1207/s15327752jpa5701_17

Preti, A., Carta, M. G., \& Petretto, D. R. (2019). Factor structure models of the SCL-90-R: Replicability across community samples of adolescents. Psychiatry Research, 272, 491-498. https://doi.org/10.1016/j.psychres.2018.12.146

Prinz, U., Nutzinger, D. O., Schulz, H., Petermann, F., Braukhaus, C., \& Andreas, S. (2013). Comparative psychometric analyses of the SCL-90-R and its short versions in patients with affective disorders. BMC psychiatry, 13(1), 104. https://doi.org/10.1186/1471-244X-13-104

Prunas, A., Sarno, I., Preti, E., Madeddu, F., \& Perugini, M. (2012). Psychometric properties of the Italian version of the SCL-90-R: A study on a large community sample. European Psychiatry, 27(8), 591-597. https://doi.org/10.1016/j.eurpsy.2010.12.006 
Radloff, L. S. (1977). The CES-D Scale:A Self-Report Depression Scale for Research in the General Population. Applied Psychological Measurement, $1(3), \quad 385-401$. https://doi.org/10.1177/014662167700100306

Sanchez, R. O. \& Ledesma, R. D. (2009). Análisis Psicométrico del Inventario de Síntomas Revisado (SCL90-R) en Población Clínica. Revista Argentina de Clinica Psicologica, 18(3), 265-274.

Sayar, K., Kirmayer, L. J., \& Taillefer, S. S. (2003). Predictors of somatic symptoms in depressive disorder. General Hospital Psychiatry, 25(2), 108-114. https://doi.org/10.1016/S0163-8343(02)00277-3

Schafer, M. H. \& Ferraro, K. F. (2013). Childhood Misfortune and Adult Health: Enduring and Cascadic Effects on Somatic and Psychological Symptoms? Journal of Aging and Health, 25(1), 3-28. https://doi.org/10.1177/0898264312464884

Schmitz, N., Hartkamp, N., Kiuse, J., Franke, G. H., Reister, G., \& Tress, W. (2000). The Symptom CheckList-90-R (SCL-90-R): A German validation study. Quality of Life Research, 9(2), 185-193. https://doi.org/10.1023/A:1008931926181

Sereda, Y. \& Dembitskyi, S. (2016). Validity assessment of the symptom checklist SCL-90-R and shortened versions for the general population in Ukraine. BMC Psychiatry, 16(1), 300. https://doi.org/10.1186/s12888-016-1014-3

Sohn, A.-R., Kim, T.-K., Ryu, E.-J., Oh, G.-J., \& Ahn, D.-H. (2010). Mental health and stress by sociodemographic characteristics among Seoul citizens. Korean Journal of Health Education Promotion, 27(1), 71-80.

Strand, B. H., Dalgard, O. S., Tambs, K., \& Rognerud, M. (2003). Measuring the mental health status of the Norwegian population: A comparison of the instruments SCL-25, SCL-10, SCL-5 and MHI-5 (SF-36). Nordic Journal of Psychiatry, 57(2), 113-118. https://doi.org/10.1080/08039480310000932

Urbán, R., Kun, B., Farkas, J., Paksi, B., Kökönyei, G., Unoka, Z., . . . Demetrovics, Z. (2014). Bifactor structural model of symptom checklists: SCL-90-R and Brief Symptom Inventory (BSI) in a non-clinical community sample. Psychiatry Research, 216(1), 146-154. https://doi.org/10.1016/j.psychres.2014.01.027

Yu, Y., Wan, C., Huebner, E. S., Zhao, X., Zeng, W., \& Shang, L. (2019). Psychometric properties of the symptom check list 90 (SCL-90) for Chinese undergraduate students. Journal of Mental Health, 28(2), 213-219. https://doi.org/10.1080/09638237.2018.1521939

Recibido 24-08-2020 | Aceptado 02-12-2020

Este trabajo se encuentra bajo una Licencia Creative Commons Atribución 4.0 Internacional que permite a terceros utilizar lo publicado siempre que se dé el crédito pertinente a los autores y a Psicodebate 